\title{
Correlation between STK33 and the pathology and prognosis of lung cancer
}

\author{
YI LU, JIE TANG, WENMEI ZHANG, CE SHEN, LING XU and DANRONG YANG \\ Department of Respiratory Medicine, Shanghai Jiao Tong University Affiliated \\ Sixth People's Hospital, Shanghai 200233, P.R. China
}

Received June 16, 2017; Accepted August 11, 2017

DOI: $10.3892 / 01.2017 .6766$

\begin{abstract}
Correlation between the expression of STK33 and the pathology of lung cancer was investigated, to explore its effects on prognosis. Hundred and two lung cancer patients diagnosed by pathological examinations were randomly selected in Shanghai Jiao Tong University Affiliated Sixth People's Hospital from February, 2012 to February, 2017 to serve as observation group, and the tumor tissues were collected. At the same time, 19 patients with lung benign lesions were selected and lung tissues were also collected to serve as control group. RT-qPCR was used to detect the expression of STK33 mRNA in tissues. Expression levels of STK33 protein were detected and compared by SP immunohistochemistry staining and western blot analysis. Statistical analysis was performed to analyze the correlation between STK33 expression and the pathology and prognosis of lung cancer. Results of PCR showed that expression level of STK33 gene in control group was significantly lower than that in observation group $(\mathrm{p}<0.05)$. The expression level of STK33 mRNA in lung adenocarcinoma and squamous cell carcinoma was lower than that in lung small cell carcinoma and large cell carcinoma $(\mathrm{p}<0.05)$. Western blot analysis showed that the expression level of STK33 protein in lung small cell carcinoma and large cell carcinoma was significantly higher than that in lung adenocarcinoma and squamous cell carcinoma $(\mathrm{p}<0.05)$. Immunohistochemistry staining showed that the positive rate of STK33 in lung large cell carcinoma (100\%) and small cell carcinoma (100\%) was significantly higher than that in lung adenocarcinoma $(88.1 \%)$ and squamous cell carcinoma $(86.2 \%)(\mathrm{p}<0.05)$. The 5 -year survival rate analysis showed that the recurrence-free survival rate and overall survival rate of STK33 gene high expression level group were significantly lower than those of low expression level group $(\mathrm{p}<0.05)$. The differential expression level of STK33 is related to the pathology and prognosis
\end{abstract}

Correspondence to: Dr Danrong Yang, Department of Respiratory Medicine, Shanghai Jiao Tong University Affiliated Sixth People's Hospital, 600 Yi Shan Road, Shanghai 200233, P.R. China

E-mail: yangdanrong17@163.com

Key words: STK33, lung cancer, pathology, prognosis of lung cancer, which is of great value in clinical diagnosis and prognosis evaluation.

\section{Introduction}

Lung cancer is one of the most serious cancers without effective treatment (1). The incidence rate of lung cancer is the highest among all types of cancers worldwide. Lung cancer also causes unacceptable high mortality rate, and accounts for $\sim 25 \%$ of the deaths caused by cancer (2). With the increased number of smokers and intensified environmental pollution, especially in industrialized cities, the incidence of lung cancer is rapidly increasing (3). At present, gene detection and targeted therapy have attracted more and more attention. Some targeted drugs have been proved to be able to prolong the life of patients with lung cancer (4). In recent years, STK33 has been shown to play an important role in the development of a variety of cancers and to participate in the regulation of DNA replication, signal transduction, cell proliferation, cell differentiation, apoptosis and tumor development. Due to the correlate STK33 and the well-known oncogene Ras, STK33 has become an active research area (5). However, the studies on the role of STK33 in the development of lung cancer are relatively rare, and the role of STK33 in carcinogenesis and development of lung cancer is still not clear $(6,7)$. This study investigated the correlation between STK33 gene expression and pathology and prognosis of lung cancer to provide reference for clinical diagnosis and treatment.

\section{Materials and methods}

Research subjects. In total 102 lung cancer patients diagnosed by pathological examinations were randomly selected in Shanghai Jiao Tong University Affiliated Sixth People's Hospital from February, 2012 to February, 2017. Tumor tissues were collected to serve as observation group. The patients included 63 males and 39 females, the age ranged from 33 to 77 years with and average age of 54.3. There were 42 cases of adenocarcinoma, 29 cases of squamous cell carcinoma, 16 cases of small cell lung cancer and 15 cases of pulmonary large cell carcinoma. At the same time, 19 patients with lung benign lesions were selected and lung tissues were also collected to serve as control group. Control group contained 8 cases of pneumonia, 5 cases of pulmonary tuberculosis, 
4 cases of benign tumors and 2 cases of lymphoid tissue atypical hyperplasia. Exclusion criteria: i) Patients with major cardiovascular and cerebrovascular diseases, and digestive disease; ii) Patients with mental disorders, or unable to communicate with researchers in a normal way; iii) Pregnant women; iii) Patients with incomplete clinical data. All patients were followed up by telephone to collect data for the analysis of 5-year survival rate. No significant differences in gender, age and other basic information were found between the two groups $(\mathrm{p}>0.05)$. The study was approved by the Ethics Committee of Shanghai Jiao Tong University Affiliated Sixth People's Hospital. All patients or their family members signed written informed consent.

Reagents. TRIzol (Life Technologies, New York, NY, USA); chloroform and isopropyl alcohol (Beijing Chemical Co., Ltd., Beijing, China); M-MLV reverse transcriptase; DNase I (both from Life Technologies); SYBR ${ }^{\circledR}$ Premix Ex Taq ${ }^{\mathrm{TM}}$ II (Takara Bio Inc., Liaoning, China); DNA Marker (TransGen Biotech, Beijing, China); primer synthesis (Beijing Genomics Institute, Guangdong, China); RIPA protein lysate (Solarbio, Beijing, China); actin and STK33 rabbit anti-human primary antibodies (Cell Signaling Technology, Boston, MA, USA); BCA kit (Life Technologies); skim milk powder (BD Biosciences, New Jersey, NY, USA); horseradish peroxidase labeled goat anti-rabbit IgG secondary antibody (Cell Signaling Technology); NC membrane (Millipore, Billerica, MA, USA); luminescent substrate kit (TransGen Biotech); SP kit (Cell Signaling Technology).

Real-time reverse transcription-quantitative polymerase chain reaction $(R T-q P C R)$. Lung cancer tissues and benign lesion tissues were collected by fiberoptic bronchoscopy and stored in liquid nitrogen before use. Tissues were ground in liquid nitrogen and TRIzol was used to extract total RNA according the instructions of the kit. The concentration of RNA samples was measured and $1 \mu \mathrm{g}$ RNA and reverse transcriptase kit were used for reverse transcription to obtain cDNA. SYBR ${ }^{\circledR}$ Premix Ex Taq ${ }^{\mathrm{TM}}$ II and cDNA were used to prepare PCR reaction system and PCR reaction was performed on Bio-Rad CFX96 qPCR Instrument to detect the expression level of STK33 in each sample. Reaction conditions are listed in Table I and primers in Table II.

Western blot analysis to quantitatively analyze the expression levels of proteins. Tissues were ground in liquid nitrogen, followed by lysis with RIPA protein lysate on ice for $30 \mathrm{~min}$. Then the samples were centrifuged $\left(12,000 \times \mathrm{g}\right.$ at $\left.4^{\circ} \mathrm{C}\right)$ for $10 \mathrm{~min}$ to collect the supernatant. The supernatant was stored in $1.5 \mathrm{ml}$ EP tube. Protein concentration was measured using BCA method, and $100 \mu \mathrm{g}$ protein from each sample was mixed with loading buffer and denatured in boiling water for $10 \mathrm{~min}$, followed by $10 \%$ sodium dodecyl sulfate-polyacrylamide gel electrophoresis (SDS-PAGE) gel electrophoresis under $40 \mathrm{~V}$ for $0.5 \mathrm{~h}$. Protein was then transferred to $\mathrm{NC}$ membrane. After blocking with $5 \%$ skim milk at $4^{\circ} \mathrm{C}$ overnight, membranes were washed 3 times (10 min each time) and incubated with primary rabbit monoclonal STK33 antibody (dilution, 1:500; cat. no. ab206296; Abcam, Cambridge, MA, USA) at room temperature for $2 \mathrm{~h}$. After washing, membranes were incubated
Table I. PCR reaction conditions

\begin{tabular}{lccr}
\hline Steps & Temperature & Time & Circle \\
\hline 1 & $94^{\circ} \mathrm{C}$ & $15 \mathrm{~min}$ & 1 \\
2 & $94^{\circ} \mathrm{C}$ & $10 \mathrm{sec}$ & 40 \\
& $50^{\circ} \mathrm{C}$ & $30 \mathrm{sec}$ & \\
& $72^{\circ} \mathrm{C}$ & $15 \mathrm{sec}$ & \\
\hline
\end{tabular}

Fluorescence recording

\begin{tabular}{llll}
\hline 3 & $72^{\circ} \mathrm{C}$ & $10 \mathrm{~min}$ & 1 \\
\hline
\end{tabular}

Table II. Primers for $\beta$-actin and STK33.

\begin{tabular}{lc}
\hline Genes & \multicolumn{1}{c}{ Primer sequences } \\
\hline$\beta$-actin & 5'-3' GTGGACATCCGCAAAGAC \\
& 3'-5' GAAAGGGTGTAACGCAACTA \\
STK33 & 5'-3' GGGAGCCAGATAAACG \\
& 3'-5' GCTTCACCCGTTAATT \\
\hline
\end{tabular}

withsecondary goat anti-rabbit (HRP) IgG antibody (dilution, 1:2,000; cat. no. ab6721; Abcam) at room temperature for $1 \mathrm{~h}$. Fluorescent substrate was then added for color development in the dark. Imaging results were quantified using ImageJ software (version X; Media Cybernetics, Silver Springs, MD, USA).

Immunohistochemistry staining to analyze the expression of proteins. SP immunohistochemistry staining was performed in strict accordance with the instructions of the kit. Determination of positive cells in stained samples: i) Brown particles; ii) color intensity is higher than the color intensity of background. Positions of observation: iii) cell cytoplasm, mainly in macrophages and tumor cells; iv) endothelial cells of interstitial tubes. Observation method: Five visual fields were randomly selected (x400). Definition of relative expression: proportion of positive cells $>50 \%, 3$ points; proportion of positive cells $25-49 \%, 2$ points; proportion of positive cells $1-24 \%, 1$ point; proportion of positive cells $<1 \%, 0$ point. Scoring according to the intensity of staining, dark color was recorded as 3 points, moderate color as 2 points, and light color as 1 point. The combination of those two scores was used as the basis for determining the expression level. Scores $\leq 3$ were recorded as low expression level, and scores $>3$ points as high expression level and positive expression.

Analysis of prognosis. A 5-year follow-up was carried out, and the recurrence-free survival rate and overall survival rate of patients with different pathologic types were recorded. Kaplan-Meier survival curve was also used to show the survival rate of patients.

Statistical analysis. All data were processed using SPSS 19.0 software (IBM, Armonk, NY, USA). Measurement data were 


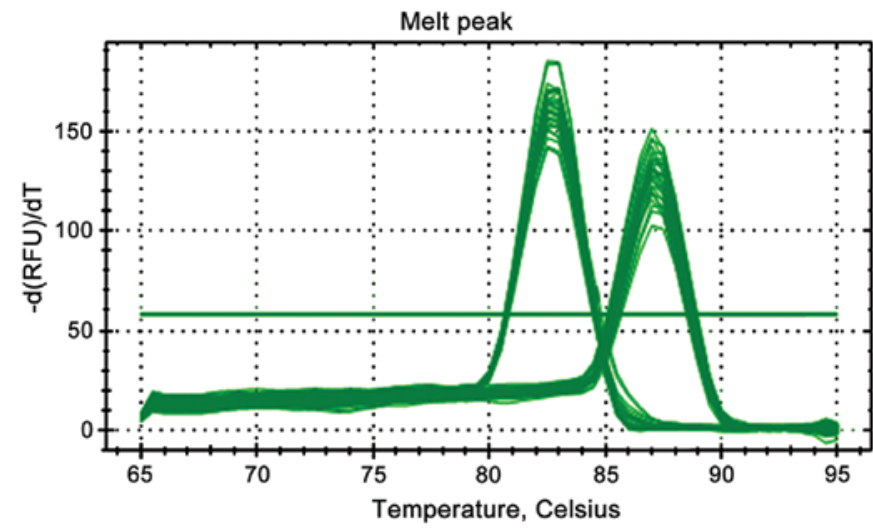

Figure 1. Melting curves of $\beta$-actin (left) and STK33 (right) PCR product. Single peaks indicate the high specificity of the primer and accuracy of results.

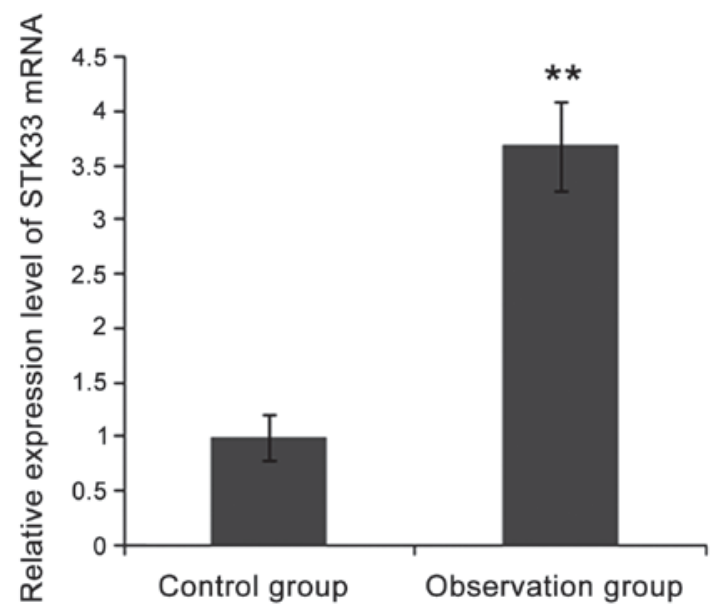

Figure 2. Expression level of STK33 mRNA in observation group and control group. RT-qPCR results showed that expression level of STK33 mRNA in observation group was significantly higher than that in control group $\left({ }^{* *} \mathrm{p}<0.01\right)$.

expressed as mean $\pm \mathrm{SD}$. Comparisons of mean values were performed by t-test and single factor analysis of variance. Count data were processed using $\chi^{2}$ test. $p<0.05$ was considered to be statistically significant.

\section{Results}

Expression of STK33 mRNA in patients with different pathological types. As shown in Fig. 1, the melting curves of $\beta$-actin and STK33 showed single peaks, indicating the high specificity of the primer and accuracy of results. As shown in Fig. 2, the expression level of STK33 mRNA in observation group was significantly higher than that in control group $(\mathrm{p}<0.05)$. As shown in Fig. 3, expression levels of STK33 mRNA in lung adenocarcinoma and squamous cell carcinoma were significantly lower than those in lung small cell carcinoma and large cell carcinoma $(\mathrm{p}<0.05)$.

Expression of STK33 protein in patients with different pathological types detected by western blot analysis. Expression level of STK33 protein in patients with four pathological types of lung cancer was significantly higher than that in patients with benign lesions $(p<0.05)$. In addition, expression level of

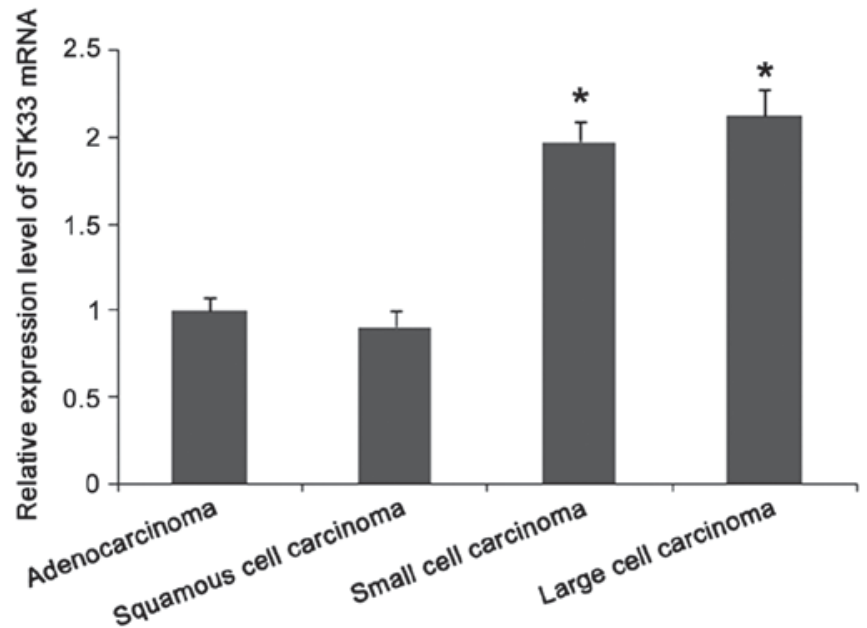

Figure 3. Expression level of STK33 mRNA in patients with different pathological types. RT-qPCR results showed that expression levels of STK33 mRNA in lung adenocarcinoma and squamous cell carcinoma were significantly lower than those in lung small cell carcinoma and large cell carcinoma $(" \mathrm{p}<0.05)$.

\section{A \\ B}
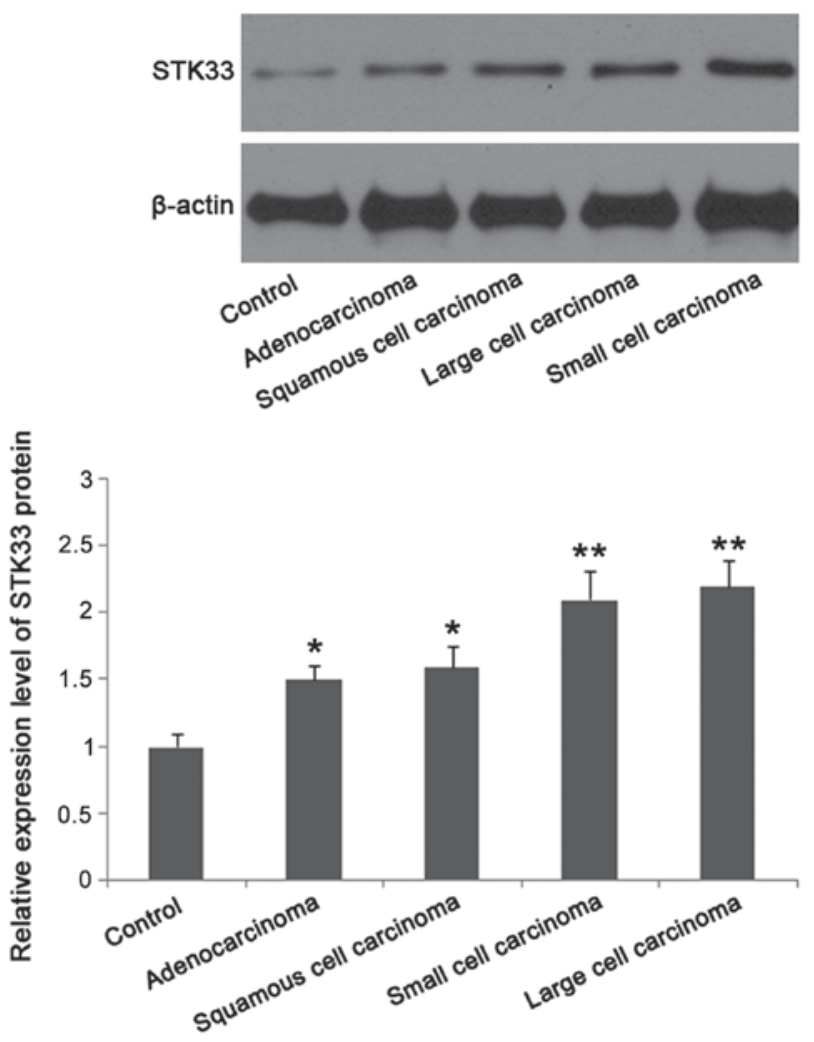

Figure 4. Detection and comparison of expression levels of STK33 protein in patients with different pathological types of lung cancer and benign lesions. (A) Representative result of western blot analysis; (B) Relative expression level of STK33 protein in patients with different pathological types of lung cancer and benign lesions. Compared with the control group, the expression level of STK33 protein in adenocarcinoma group and squamous cell carcinoma group was significantly increased ( $\mathrm{p}<0.05)$. Compared with adenocarcinoma group, expression level of STK33 protein was significantly increased in small cell carcinoma group and in the large cell carcinoma group increased $(* \mathrm{p}<0.01)$

STK33 protein in lung small cell carcinoma and large cell carcinoma was significantly higher than that in lung adenocarcinoma and squamous cell carcinoma $(\mathrm{p}<0.05)$ (Fig. 4). 
Table III. Results of immunohistochemistry staining of lung cancer tissue $[\mathrm{n}(\%)]$.

\begin{tabular}{|c|c|c|c|c|c|}
\hline Types & Cases & Negative & Positive & & P-value \\
\hline Adenocarcinoma & 42 & $5(11.9)$ & $37(88.1)$ & 12.653 & $3<0.001$ \\
\hline $\begin{array}{l}\text { Squamous cell } \\
\text { carcinoma }\end{array}$ & 29 & $4(13.8)$ & $25(86.2)$ & 14.823 & $3<0.001$ \\
\hline Large cell carcinoma & 15 & $0(0)$ & $15(100)$ & & \\
\hline Small cell carcinoma & 16 & $0(0)$ & $16(100)$ & & \\
\hline
\end{tabular}

Table IV. Comparison of survival rate between STK33 gene high expression group and low expression group [n (\%)].

\begin{tabular}{lccc}
\hline Groups & \multicolumn{2}{c}{$\begin{array}{c}\text { Recurrence-free } \\
\text { survival rate }\end{array}$} & $\begin{array}{c}\text { Overall } \\
\text { survival rate }\end{array}$ \\
\hline Low expression group & 71 & $34(47.9)$ & $50(70.4)$ \\
High expression group & 31 & $7(22.6)$ & $8(25.8)$ \\
$\chi^{2}$ & & 13.92 & 39.841 \\
P-value & & $<0.001$ & $<0.001$ \\
\hline
\end{tabular}

Expression of STK33 protein detected by immunohistochemistry staining. Immunohistochemistry staining showed that the positive rate of STK33 in lung large cell carcinoma (100\%) and small cell carcinoma (100\%) was significantly higher than that in lung adenocarcinoma (88.1\%) and squamous cell carcinoma (86.2\%), $(\mathrm{p}<0.05)$ (Table III).

Correlation between STK33 expression and prognosis of lung cancer patients. According to the results of immunohistochemistry staining, negative expression group is the low expression group, and positive expression group is the high expression group. The 5-year survival rate analysis showed that the recurrence-free survival rate and overall survival rate of STK33 gene high expression group were significantly lower than those of low expression group $(\mathrm{p}<0.05)$ (Table IV). Kaplan-Meier survival curves of the two groups are shown in Fig. 5 .

\section{Discussion}

Lung, as the hub for the gas exchange between the human body and outside world, can easily be affected by environment. Lesions or even cancer can develop more easily in lung than other organs. Lung cancer cells can easily migrate, and the cure rate is very low. With the development of molecular biology, the diagnosis and treatment of cancer has moved from chemotherapy and radiotherapy to individualized therapies at molecular level, among which the emergence of targeted small molecule drugs for different genes of different cancers is a typical representative $(8,9)$. Lung cancer-related genes of interest include EGFR, KRAS, and BRAF, and targeted drugs have also been developed to target the genes (10-12). However, with the deepening of research and the gradual mining of related molecular signaling pathways, more and more lung cancer-related genes, such as STK33, have been identified (13).

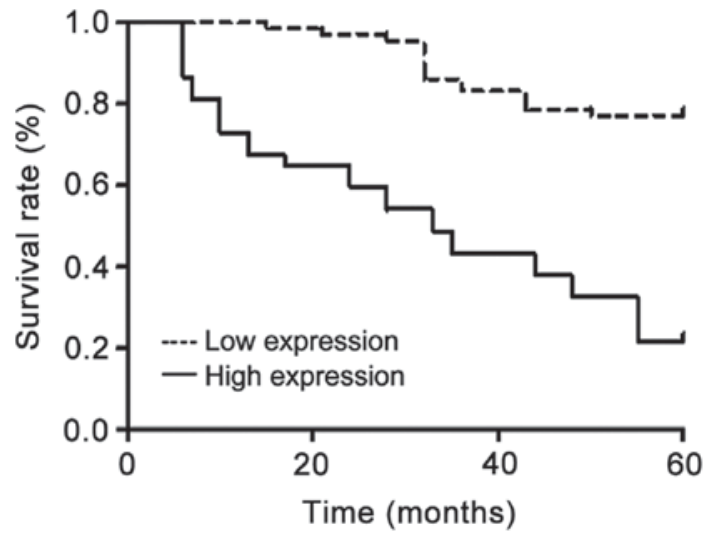

Figure 5. Kaplan-Meier survival curves show the 5-year survival of lung cancer patients. The overall survival rate of STK33 high expression group was significantly higher than that of low expression group $(\mathrm{p}<0.01)$.

STK33 is located in the human chromosome 11p15.3 region. Studies have shown that multiple genes in this region were associated with the occurrence and development of a variety of clinical diseases, and the functional abnormalities of the genes can led to the occurrence of tumors (14). STK33 gene encodes a novel serine/threonine protein kinase. Due to the specific structure, STK33 cannot only activate the protease but also participate in a variety of life activities by affecting absorption of calcium (15). STK33, as a newly discovered gene with unclear mechanism, has attracted increasing attention.

STK33 can indirectly interact with Ras gene to cause synthetic lethality of various cancer cells (16). Using RNAi, Scholl et al have demonstrated that STK33 gene and KRAS gene are two independent and indispensable genes that affect the occurrence, growth and metastasis of cancer cells (17). Some researchers believe that STK33 can participate in agglutination of mesenchymal cells through specific phosphorylated proteins to cause changes in cell structure, affect normal physiological function, and promote the activation and proliferation of tumor cells (18). Related studies also showed that the presence of STK33 protein in colon cancer, lung cancer, breast cancer, pancreatic cancer and other tumor cells is closely related to occurrence of mutations in KRAS gene $(19,20)$. However, the mechanism of the role of STK33 in the pathogenesis of cancer, especially lung cancer is still unclear.

In this study, the correlation between the expression of STK33 gene and the clinicopathological features of lung cancer was investigated, and the effects of the expression of STK33 on the prognosis of lung cancer were also explored. Results showed that the expression of STK33 gene was closely correlated with the pathologic types of lung cancer, and significant differences were found in expression level of STK33 protein among patients with different pathologic types. Expression level of STK33 gene in lung cancer group was significantly higher than that in benign lesion group $(\mathrm{p}<0.05)$. Expression level of STK33 mRNA in lung adenocarcinoma and squamous cell carcinoma was significantly lower than that in lung small cell carcinoma and large cell carcinoma $(\mathrm{p}<0.05)$. Western blot analysis showed that the expression level of STK33 protein in lung small cell carcinoma and large cell carcinoma was significantly higher than that in lung adenocarcinoma and 
squamous cell carcinoma $(\mathrm{p}<0.05)$. Immunohistochemistry staining showed that the positive rate of STK33 in lung large cell carcinoma (100\%) and small cell carcinoma (100\%) was significantly higher than that in lung adenocarcinoma $(88.1 \%)$ and squamous cell carcinoma $(86.2 \%)(\mathrm{p}<0.05)$. All the data indicate that STK33 can potentially be used as a biomarker of non-cancerous lesions. The 5-year survival rate analysis showed that the recurrence-free survival rate and overall survival rate of STK33 gene high expression group were significantly lower than those of low expression group $(\mathrm{p}<0.05)$, indicating that this gene is closely correlated with the degree and clinical staging of lung cancer.

In conclusion, the differential expression level of STK33 is correlated with the pathology and prognosis of lung cancer, which is of great value in clinical diagnosis and prognosis evaluation.

\section{Acknowledgements}

This study was supported by the Natural Science Foundation of Shanghai Jiao Tong University Affiliated Sixth People's Hospital (1575).

\section{References}

1. Wakeam E, Acuna SA, Leighl NB, Giuliani ME, Finlayson SRG, Varghese TK and Darling GE: Surgery versus chemotherapy and radiotherapy for early and locally advanced small cell lung cancer: A propensity-matched analysis of survival. Lung Cancer 109: 78-88, 2017.

2. Lopez-Pastorini A, Riedel R, Koryllos A, Beckers F, Ludwig C and Stoelben E: The impact of preoperative elevated serum C-reactive protein on postoperative morbidity and mortality after anatomic resection for lung cancer. Lung Cancer 109: 68-73, 2017.

3. Wu L, Leng D, Cun D, Foged C and Yang M: Advances in combination therapy of lung cancer: Rationales, delivery technologies and dosage regimens. J Control Release 260: 78-91, 2017.

4. Chen C, Huang L, Zhang G, Li Y, Li L, Bai X, Liu W, Wang H and Li J: STK33 potentiates the malignancy of hypopharyngeal squamous carcinoma: Possible relation to calcium. Cancer Biol Ther 17: 976-984, 2016.

5. Azoitei N, Hoffmann CM, Ellegast JM, Ball CR, Obermayer K, Gößele U, Koch B, Faber K, Genze F, Schrader M, et al: Targeting of KRAS mutant tumors by HSP90 inhibitors involves degradation of STK33. J Exp Med 209: 697-711, 2012.

6. Wang P, Cheng H, Wu J, Yan A and Zhang L: STK33 plays an important positive role in the development of human large cell lung cancers with variable metastatic potential. Acta Biochim Biophys Sin (Shanghai) 47: 214-223, 2015.
7. Brauksiepe B, Baumgarten L, Reuss S and Schmidt ER: Co-localization of serine/threonine kinase 33 (Stk33) and vimentin in the hypothalamus. Cell Tissue Res 355: 189-199, 2014.

8. Milenic DE, Baidoo KE, Kim YS, Barkley R and Brechbiel MW: Targeted $\alpha$-particle radiation therapy of HER1-positive disseminated intraperitoneal disease: An investigation of the human anti-EGFR monoclonal antibody, panitumumab. Transl Oncol 10: 535-545, 2017.

9. Groner B and von Manstein V: Jak Stat signaling and cancer: Opportunities, benefits and side effects of targeted inhibition. Mol Cell Endocrinol 451: 1-14, 2017.

10. Zhu Y, Bassoff N, Reinshagen C, Bhere D, Nowicki MO, Lawler SE, Roux J and Shah K: Bi-specific molecule against EGFR and death receptors simultaneously targets proliferation and death pathways in tumors. Sci Rep 7: 2602, 2017.

11. Shen H, Xing C, Cui K, Li Y, Zhang J, Du R, Zhang X and Li Y: MicroRNA-30a attenuates mutant KRAS-driven colorectal tumorigenesis via direct suppression of ME1. Cell Death Differ 24: 1253-1262, 2017.

12. Lim SY, Menzies AM and Rizos H: Mechanisms and strategies to overcome resistance to molecularly targeted therapy for melanoma. Cancer 123 (S11): 2118-2129, 2017.

13. Ye H, Shao M, Shi X, Wu L, Xu B, Qu Q and Qu J: Predictive assessment in pharmacogenetics of glutathione $\mathrm{S}$-transferases genes on efficacy of platinum-based chemotherapy in non-small cell lung cancer patients. Sci Rep 7: 2670, 2017.

14. Brace PT, Tezera LB, Bielecka MK, Mellows T, Garay D, Tian S, Rand L, Green J, Jogai S, Steele AJ, et al: Mycobacterium tuberculosis subverts negative regulatory pathways in human macrophages to drive immunopathology. PLoS Pathog 13: e1006367, 2017.

15. Reuss S, Brauksiepe B, Disque-Kaiser U and Olivier T: Serine/threonine-kinase 33 (Stk33) - Component of the neuroendocrine network? Brain Res 1655: 152-160, 2017.

16. Huang L, Chen C, Zhang G, Ju Y, Zhang J, Wang H and Li J: STK 33 overexpression in hypopharyngeal squamous cell carcinoma: Possible role in tumorigenesis. BMC Cancer 15: 13, 2015.

17. Scholl C, Fröhling S, Dunn IF, Schinzel AC, Barbie DA, Kim SY, Silver SJ, Tamayo P, Wadlow RC, Ramaswamy S, et al: Synthetic lethal interaction between oncogenic KRAS dependency and STK33 suppression in human cancer cells. Cell 137: 821-834, 2009.

18. Mujica AO, Hankeln T and Schmidt ER: A novel serine/threonine kinase gene, STK33, on human chromosome 11p15.3. Gene 280: 175-181, 2001.

19. Yang T, Song B, Zhang J, Yang GS, Zhang H, Yu WF, Wu MC, Lu JH and Shen F: STK33 promotes hepatocellular carcinoma through binding to c-Myc. Gut 65: 124-133, 2016.

20. Babij C, Zhang Y, Kurzeja RJ, Munzli A, Shehabeldin A, Fernando M, Quon K, Kassner PD, Ruefli-Brasse AA, Watson VJ, et al: STK33 kinase activity is nonessential in KRAS-dependent cancer cells. Cancer Res 71: 5818-5826, 2011. 